Forschende

Komplementärmedizin und

Klassische Naturheilkunde

\title{
Economy Chased Medicine: Ökonomisierung der Gesundheit - Chance oder Bedrohung für die Komplementärmedizin?
}

\author{
Harald Walach \\ Universitätsklinikum, Institut für Umweltmedizin und Krankenhaushygiene, Freiburg i.Br., Deutschland
}

\begin{abstract}
Dieser Tage ist mir ein Erlebnis aus meiner Jugend in den Sinn gekommen: Wir hatten mit Schlauchbooten eine Flussfahrt auf dem Lech organisiert, einem Fluss, der durch unzählige Wehre gestaut und gebremst wird. Manche Wehre mussten wir, die Boote tragend, umgehen. Bei einigen kleineren versuchten wir durchzufahren. Es gelang uns auch bis auf ein einziges Mal. Da war die Rückstromwalze am Fuss des Wehres so stark, dass sie das Boot gefangen hielt und wir nicht mehr freikamen. Langsam drehte sich das Boot parallel zur Wasserwalze, wurde dann vom fallenden Wasser erfasst und kenterte. Einen Moment nur versuchte ich, gegen den mächtigen Strom anzukämpfen, gab aber rasch auf. Ich liess mich vom Wasser hinunterziehen bis auf den Grund und konnte unten mit dem strömenden Wasser abtauchen. Etwa $20 \mathrm{~m}$ von der Walze entfernt wurde ich wieder ausgespuckt. Diese Episode hat mir eine wichtige Lehre erteilt: gegen mächtige Strömungen anzukämpfen ist Irrsinn. Man kann nur ihre Kraft nutzen, ihnen so lange folgen, bis sich eine Möglichkeit bietet freizukommen.
\end{abstract}

Eine solche mächtige Strömung, gegen die sich zu stellen sinnlos wäre, ist die globale Ökonomisierung, die auch vor der Gesundheit nicht Halt macht. In Deutschland hat diese Tendenz ihre Maximen langsam, fast unmerklich auch ins Gesundheitswesen sickern lassen. In der Schweiz ist ein fulminanter Auftakt bereits durch die Presse gegangen: Bundesrat Pascal Couchepin, der liberale Innenminister und als solcher in der Schweiz auch für das Gesundheitswesen zuständig, hat beschlossen, alle komplementärmedizinischen Verfahren aus der Grundversorgung zu streichen. Damit ist das mit viel Spannung erwartete Ergebnis des so genannten «Programmes Evaluation Komplementärmedizin», kurz PEK, eines 7-jährigen, einmaligen Feldversuchs zur Nützlichkeit der Komplementärmedizin in der niedergelassenen Versorgung, durch eine politische Entscheidung bagatellisiert worden.

Couchepins Agenda ist an sich lobenswert und verständlich: Die Kosten im Schweizer Gesundheitswesen sind hoch und müssen gesenkt werden. Dies kann nur durch drei mögliche Prozesse geschehen: (1) Die Anbieter senken ihre Preise und arbeiten kostengünstiger; dies würde zu einer Einkommensreduktion im Gesundheitswesen führen und ist wohl kaum realistisch. (2) Die Konsumenten senken ihre Ansprüche; angesichts der wachsenden Anspruchshaltung wohl kaum zu erwarten. (3) Leistungen, die nicht absolut notwendig sind, müssen privat bezahlt werden.
Damit wird die Zwei- oder Drei-Klassenmedizin explizit zum Standard. Diese Lösung ist wahrscheinlich die in Zukunft zu erwartende.

Genau dies ist nun in der Schweiz geschehen. Die Komplementärmedizin fällt der ökonomischen Vernunft zuerst zum Opfer. Einige Kommentatoren der Schweizer Presse haben zu Recht gesehen, dass das Schweizer Department des Inneren damit den Stier nicht bei den Hörnern gepackt hat, sondern vorsichtigerweise erst einmal in einem unbemerkten Augenblick ein Schwanzhärchen abgeschnitten hat, um zu testen, wie er reagiert. Die Vermutung, so der Kommentar, könnte sein, dass über eine öffentliche Diskussion gerade des belanglosesten Steinchens im Mosaik der Kosten eine allgemeine Bereitschaft zum Einsparen auch anderswo erzeugt wird.

Eine kurze Retrospektive und Analyse der Prozesse ist lehrreich: Vor etwa 8 Jahren hatte Couchepins Vorgängerin, Ruth Dreyfuss, auf vielseitigen Wunsch angekündigt, die Komplementärmedizin in die Grundversorgung aufzunehmen und in einem einmaligen, nationalen Versuch 7 Jahre lang zu evaluieren, wie sich Kosten und Gesundheit entwickeln. Über einige Jahre geschah nach aussen sichtbar gar nichts. Eine Arbeitsgruppe hatte sich gebildet, die sich erst einmal Gedanken machen musste, wie man denn vorgehen solle. Erst 3 Jahre, nachdem das PEK offiziell begonnen wurde, erfolgte die Ausschreibung, still und leise zwischen den Jahren. Für die eigentliche Durchführung der Evaluationsforschung standen nun gerade noch 3,5 Jahre zur Verfügung. Das muss wohl der Grund dafür gewesen sein, dass nur etwa 2 Wochen Zeit für die Einreichung von Vorschlägen war. Mit der Evaluation wurde dann eine Berner Gruppe betraut, die zwar viel Erfahrung mit Outcome-Forschung, aber keine Erfahrung mit Komplementärmedizin hatte. Ein weiteres Jahr verstrich, bevor irgendwelche Daten erhoben werden konnten. Gut vorbereitete Projekte, etwa das der Homöopathen, einen randomisierten Praxisvergleich durchzuführen, wurden gekippt. Bislang sind die Daten der durchgeführten Studien bis auf Ausnahmen [Busato, persönliche Mitteilung] nicht in einem begutachteten Organ publiziert. Die Originaldokumente stehen mittlerweile auf der Homepage des Bundesamtes für Gesundheit (www.bag.admin. ch/kv/forschung/d/2005). Die eigentliche Comedia dell' Arte kommt aber noch:

Die abschliessende Sitzung des wissenschaftlichen Beirats, der die Protokolle begutachtet und das PEK wissenschaftlich begleitet

\begin{tabular}{ll}
\hline KARGER & ๑ 2005 S. Karger GmbH, Freiburg \\
$\begin{array}{l}\text { Fax +49 76145207 14 } \\
\begin{array}{l}\text { E-mail Information@Karger.de } \\
\text { www.karger.com }\end{array}\end{array}$ & $\begin{array}{l}\text { Accessible online at: } \\
\text { www.karger.com/fkm }\end{array}$
\end{tabular}

Prof. Dr. Dr. Harald Walach

Universitätsklinikum

Institut für Umweltmedizin und Krankenhaushygiene

Hugstetterstr. 55, 79106 Freiburg, Deutschland

Tel. +49 761 270-5497, Fax -7224

E-mail harald.walach@uniklinik-freiburg.de 
hatte, wurde kurzerhand abgesagt. Eine Diskussion der Ergebnisse vor der Entscheidung sollte anscheinend vermieden werden. Ich habe mich dann als Mitglied des Review Boards mit dem stellvertretenden Direktor des Bundesamts für Gesundheit in Verbindung gesetzt und erhielt die Auskunft, die Entscheidung über den Verbleib der Komplementärmedizin in der Grundversorgung sei eine rein politische (das finde ich akzeptabel). Deshalb wäre eine Diskussion der wissenschaftlichen Ergebnisse für die Entscheidung irrelevant, und die wissenschaftlichen Daten «Abfallprodukt». Das finde ich inakzeptabel. Eine Intervention über die Presse hat dazu geführt, dass der Beirat des PEK doch noch über die Ergebnisse diskutieren darf, sozusagen post festum. Immerhin.

Das Vorgehen macht Folgendes sichtbar: Wissenschaftliche Daten sind in diesem Interessengeflecht nur von begrenzter Bedeutung. Damit sind Bruno Latours Analysen des Wissenschaftsprozesses [1] bestätigt. Der Wissenschaftler bzw. das wissenschaftliche Kollektiv sind nur ein Mitspieler. Wichtige soziale und politische Interessengruppen sind mindestens ebenso wichtig. Nicht die Abbildung der Wirklichkeit, sondern die Durchsetzung von Interessen mit oder ohne die Mittel der Wissenschaft ist zentral [2]. Das politische Programm auf der Agenda ist kurzfristiges Einsparen und Kosten senken. Ginge es um reale, langfristige Einsparungen, hätte man die Kostendaten des PEK öffentlich diskutiert und dann entsprechend entschieden. (Einige haben versucht, durch eine Vorab-Publikation von Befunden zu Kosten dämpfenden Effekten der Komplementärmedizin eine solche öffentliche Diskussion in Gang zu bringen. Vergeblich) Nein. Im Vordergrund steht die kurzfristige Einsparung. Tausend Ärzte weniger, die an der Grundversorgung beteiligt sind und sich stattdessen privat finanzieren, bedeutet mehr Geld für die anderen, weniger direkte und offensichtliche Kosten für die Kassen.

Diese Situation lehrt uns aber noch mehr: In Zukunft wird die Wirksamkeitsdebatte immer weniger wichtig werden. Der leicht vorauszusehende Entscheidungsfall wird nicht mehr lauten: Wirkt eine Intervention, und wie viel kostet sie? Sondern: Wie viel kostet eine Intervention, und können wir sie uns leisten? Die Dialektik des beginnenden Prozesses wird unweigerlich dazu führen, dass die allseits geführte Debatte um «Daten gestützte Entscheidung in der Medizin», wie ich «evidence based medicine» gerne übersetzt sehen würde, in wenigen Jahren vollkommen von der «Kosten gesteuerten Entscheidung in der Medizin» («cost chased medicine») überlagert werden wird. Die Reihung der Fragen wird sich also, verglichen mit heute, vermutlich umkehren.

Dies kann bei allem Lamento eine grosse Chance bedeuten. Wenn sich nämlich belegen lässt, dass Komplementärmedizin vergleichsweise kostengünstig arbeitet, vergleichsweise hohe Zufriedenheit bei akzeptablem oder sogar gutem Ergebnis produziert, dann wird kaum mehr einer fragen, ob nicht vielleicht doch alles Plazebo ist. Bald wird sich nämlich zeigen: Das meiste, was wirkt, wirkt so, dass es die Selbstheilungskräfte auf die eine oder andere Art anregt, so dass der Organismus von selbst zur Gesundheit zurückfindet. Eine etwas höflichere und präzisere Beschreibung dessen, was sich hinter dem Begriff «Plazebo» verbirgt [3]. Dann wird die Frage allenfalls sein: Welches Plazebo ist günstiger, nachhaltiger, nebenwirkungsfreier und beliebter?

Insofern ist es vermutlich nützlich, wenn wir das tun, was die Schweizer Politik bislang zu verhindern versucht hat: gute Daten vorzulegen für die allseits bekannte, aber bis auf erste Versuche [4-11] sehr schlecht belegte Erfahrung, dass Komplementärmedizin günstig ist, langfristig jedenfalls, dass sie beliebt ist, und dabei auch noch vergleichsweise gut hilft. Ein Verteilungskampf hat begonnen. Der Kampfbegriff war bislang der der «Wirksamkeit». Das Anathema hiess: «Plazebo». Mittlerweile heisst der Kampfbegriff: «Kostensenkung» und das Anathema ist: «Kosten treibend». Während die Wirksamkeitsdebatte von der Komplementärmedizin nicht leicht zu gewinnen ist, und zwar aus theoretischen Gründen [12, 13], ist die Kostendebatte leichter zu gewinnen. Die Lektion aus den jüngsten Ereignissen muss sein: die Kraft der Strömung nutzen, statt gegen sie zu kämpfen, und am besten gleich die Energie der Strömung für die eigenen Ziele einsetzen.

\section{Dank}

Meine Arbeit wird vom Samueli Institute gefördert. Ein spezieller Dank an Pascal Couchepin und Hans Heinrich Brunner für die Einsichten, die sie mir vermittelt haben.

\section{Literatur}

1 Latour B: Die Hoffnung der Pandora: Untersuchungen zur Wirklichkeit der Wissenschaften. Frankfurt, Suhrkamp, 2000

$\checkmark 2$ Walach H: Wider naiven Empirismus und verkleidete Machtansprüche - politische und wissenschaftstheoretische Splitter. Forsch Komplementärmed Klass Naturheilkd 2004;11:72-75.

$\checkmark 3$ Walach H, Jonas WB: Placebo research: The evidence base for harnessing self-healing capacities. J Altern Complement Med 2004;10:S103-S112.

4 Moebus S, Hirche H, Jöckel K-H: Naturmedizin und Therapiekosten: Erste Ergebnisse einer Machbarkeitsstudie. Erste Ergebnisse einer Langzeitbeobachtungsstudie zur Erprobungsregelung nordrhein-westfälischer Betriebskrankenkassen. Naturamed 1998;13:16-25.

5 Moebus S, Hirche H, Ose C, Jöckel K-H: Results of an observational pilot study on the effects of nonconventional therapies - a pre/post comparison and cost outcome analysis; in Abel U, Koch A (eds) Nonrandomized Comparative Clinical Studies. Düsseldorf, Symposion Publishing, 1998, pp 15-25.
6 Güthlin C, Lange O, Walach H: Measuring the effects of acupuncture and homoeopathy in general practice: An uncontrolled prospective documentation approach. BMC Public Health 2004;4:6.

7 Becker-Witt C, Lüdtke R, Weisshuhn TER, Willich $\mathrm{SN}$ : Diagnoses and treatment in homeopathic medical practice. Forsch Komplementärmed Klassische Naturheilkd 2004;11: 98-103.

8 Becker-Witt C, Keil T, Roll S, et al: Effectiveness and costs of homeopathy compared to conventional medicine - a prospective multicenter cohort study; in: Improving the Success of Homeopathy 4: Bridging the Credibility Gap. London, The Royal London Homeopathic Hospital, 2003; pp 57-60.

$\checkmark$ Brinkhaus B, Becker-Witt C, Jena S, et al: Acupuncture randomized trials (ART) in patients with chronic low back pain and osteoarthritis of the knee - design and protocols. Forsch Komplementärmed Klass Naturheilkd 2003;10:185-191.
10 Hamre HJ, Becker-Witt C, Glockmann A, Ziegler R, Willich SN, Kiene H: Anthroposophic therapies in chronic diesease: The Anthroposophic Medicine Outcome Study (AMOS). Eur J Med Res 2004; 9:351-360.

11 Melchart D, Linde K, Streng A, et al: Acupuncture randomized trials (ART) in patients with migraine or tension-type headache - design and protocols. Forsch Komplementärmed Klass Naturheilkd 2003; 10:179-184.

12 Walach H: Entanglement model of homeopathy as an example of generalizsed entanglement predicted by Weak Quantum Theory. Forsch Komplementärmed Klass Naturheilkd 2003:10:192-200.

13 Walach H: Generalised entanglement and its relevance for complementary and alternative Medicine. J Altern Complement Med 2005; in print. 